\title{
Thermodynamics of an accelerated expanding universe
}

\author{
Bin Wang* \\ Department of Physics, Fudan University, \\ Shanghai 200433, People's Republic of China \\ Yungui Gongt \\ Institute of Applied Physics and College of Electronic Engineering, \\ Chongqing University of Posts and Telecommunications, Chongqing 400065, China \\ Elcio Abdallat \\ Instituto de Fisica, Universidade de Sao Paulo, \\ C.P.66.318, CEP 05315-970, Sao Paulo, Brazil
}

\begin{abstract}
We investigate the laws of thermodynamics in an accelerating universe driven by dark energy with a time-dependent equation of state. In the case we consider that the physically relevant part of the Universe is that envelopped by the dynamical apparent horizon, we have shown that both the first law and second law of thermodynamics are satisfied. On the other hand, if the boundary of the Universe is considered to be the cosmological event horizon the thermodynamical description based on the definitions of boundary entropy and temperature breaks down. No parameter redefinition can rescue the thermodynamics laws from such a fate, rendering the cosmological event horizon unphysical from the point of view of the laws of thermodynamics.

PACS numbers: $98.80 . \mathrm{Cq}$; $98.80 .-\mathrm{k}$
\end{abstract}

*Electronic address: wangb@fudan.edu.cn

${ }^{\dagger}$ Electronic address: gongyg@cqupt.edu.cn

${ }^{\ddagger}$ Electronic address: eabdalla@fma.if.usp.br 


\section{INTRODUCTION}

Numerous and complementary cosmological observations tell us that our universe is experiencing today an accelerated expansion [1]. From the Wilkinson Microwave Anisotropic Probe (WMAP) results, such an acceleration is driven by a so-called dark energy (DE) 2]. Such a new element of the universe, capable of accelerating, must, in accordance with the Friedman equation (unless we adopt wide modifications of Einstein gravity), have a pressure less than minus one third of the energy density. In view of the large reinterpretation of concepts implied by this energy it has been even baptised under the suggesting name of quintessence.

The DE has been sought within a wide range of physical phenomena, including a cosmological constant whose equation of state is $P / \rho \equiv w_{D}=-1$, the above mentioned quintessence with $-1<w_{D}<-1 / 3$, or an exotic field with $w_{D}<-1$ [3] , which can lead to even more strange and undesirable effects such as a Big Rip, a future unavoidable singularity of space-time. An equation of state which gradually changes with the cosmological time is also a realistic possibility to explain the acceleration [4, 5, 6]. Except for the so far well known fact that DE has a negative pressure, the nature of this energy component still remains a complete mystery.

In this new conceptual set up, one of the important questions concerns the thermodynamical behaviour of an accelerated expanding universe driven by a DE. Some recent discussions on this topic can be found in [7]-[10]. With the cosmological constant, the universe is an ethernally accelerating de Sitter (dS) space.

On the other hand, defining a quantum theory for General Relativity turns out to be a problem closely related to the connection between General Relativity itself and thermodynamics. The first such hint has been obtained long ago by Bekenstein 11] who outlined the laws of thermodynamics in the presence of Black Holes which turned out to be an equivalent to the laws of Black Holes mechanics [12]. Einstein Equation can even be derived from the proportionality of entropy and horizon area (among some further technical details) 13]. In recent years, Black Holes entropy was used as a means to a new reformulation of gravity in terms of the holographic principle [14]. Relations between the entropy in the so called gravity bulk and a boundary Conformal Field Theory have been derived as a consequence of such a relation [15], promoting such ideas as the most influencial in recent years' Quantum Field Theory.

The thermodynamics corresponding to an accelerated de Sitter Universe was studied some years ago [16]. There is a cosmological event horizon, analogous to a black hole horizon, which can be associated with thermodynamical variables. Supposing that some energy passes through the 
cosmological event horizon, the definitions of Black Hole temperature and entropy imply that the first law of thermodynamics is valid. Following earlier work, quantum gravity in de Sitter space has been related to a Conformal Field Theory on the space-like boundary of de Sitter space [17, 18]. It has been argued that the bulk/boundary relation might be a consequence of general aspects of Quantum Field Theory [19].

For the accelerating universe driven by quintessence with constant pressure to energy densities $w_{D}$ in the range $-1<w_{D}<-1 / 3$, which is called Q-space in 7], Bousso argued that a thermodynamical description is approximately valid and he has shown shown that the first law of thermodynamics holds at the apparent horizon of the Q-space. The first law has also been derived on the apparent horizon of FRW universe with spatial curvatures [20]. There is a subtlety between the definitions of the apparent horizon and of the event horizon of the universe. In the dS universe they degenerate, while they are separated entities in quasi-dS universes. The question one may raise is whether the first law holds for both, the cosmological event horizon and the apparent horizon, with the definitions of temperature and entropy that we find in the de Sitter horizon. Further, when the DE equation of state is time-dependent, especially with the transition from $w_{D}>-1$ to $w_{D}<-1$ as indicated by recent observation [4], it would be interesting to investigate the first law of thermodynamics, or the entropy and temperature definitions at cosmological horizons.

The study of the first law of thermodynamics in the accelerated expanding universe serves as the first motivation of the present paper. Besides we are also going to study the expression of the entropy enveloped by cosmological horizons and examine the second law of thermodynamics derived from such a definition for an accelerating universe. Our attention will be focused on the accelerating universe driven by DE with the transition equation of state described in the recent model [5].

\section{THE FIRST LAW OF THERMODYNAMICS}

In this section we investigate the basic thermodynamic properties of accelerating universes. such as entropy, energy and temperature. We examine the first law of thermodynamics on the apparent horizon as well as on the cosmological event horizon. We begin by studying the thermodynamics of the Q-space with constant equation of state for DE, that is, $-1<w_{D}<-1 / 3$. 


\section{A. Q-space with constant equation of state for the DE $\left(-1<w_{D}<-1 / 3\right)$.}

The dynamical evolution of the scale factor and the matter density is determined by the Einstein equations, which can be written in the form

$$
(\dot{a} / a)^{2}=8 \pi \rho / 3, \quad \ddot{a} / a=-4 \pi(\rho+3 P) / 3,
$$

where the DE energy density $\rho$ and the pressure $P$ obey $P=w_{D} \rho$.

Defining $\epsilon=3\left(w_{D}+1\right) / 2$, for a constant equation of state we have $a(t)=t^{1 / \epsilon}$ and $\rho(t)=$ $3 /\left(8 \pi \epsilon^{2} t^{2}\right)$, where $0<\epsilon<1\left(-1<w_{D}<-1 / 3\right)$. This set describes the accelerating Q-space [7], For $\epsilon>1$ we the decelerating universe while $\epsilon=0$ corresponds to the de Sitter space.

The event horizon for the Q-space is $R_{E}=a \int_{t}^{\infty} d t / a=-\epsilon t /(\epsilon-1)$. The apparent horizon reads $R_{A}=1 / H=\epsilon t$. The horizons do not differ much, they relate by $R_{A} / R_{E}=1-\epsilon$. Except for the phantom universe, $w<-1$, which we have to avoid for a constant equation of state in view of the Big Rip (we actually avoid this case here), the apparent horizon is smaller than the event horizon by a fixed ratio close to unity. Neither the event horizon nor the apparent horizon changes significantly over one Hubble time, $t_{H} \dot{R_{X}} / R_{X}=\epsilon<1, X=A, E$. It is thus possible to use the equilibrium thermodynamical theory here []], especially for a (very) accelerated phase. We shall take, as in the black hole case,

$$
S_{X}=\pi R_{X}^{2}, \quad T_{X}=1 /\left(2 \pi R_{X}\right) \quad X=A, E,
$$

to describe the temperature of the horizon and the entropy.

Now let us examine the first law of thermodynamics on these two horizons. For the apparent horizon the first law was obtained in 7]. The amount of energy crossing the apparent horizon during the time interval $d t$ is

$$
-d E=4 \pi R_{A}^{2} T_{a b} k^{a} k^{b} d t=4 \pi R_{A}^{2} \rho(1+w) d t=\epsilon d t
$$

The apparent horizon entropy increases by the amount

$$
d S_{A}=\left(2 \pi R_{A}\right) \dot{R}_{A} d t=\left(2 \pi R_{A}\right) \epsilon d t
$$

Comparing (3) with (4) and using the definition of the temperature, the first law on the apparent horizon, $-d E=T_{A} d S_{A}$, was confirmed.

We now work with the cosmological event horizon. The total energy flow through the event horizon can be similarly got as

$$
-d E=4 \pi R_{E}^{2} \rho(1+w) d t=\epsilon d t /(1-\epsilon)^{2} .
$$


The entropy of the event horizon increases by

$$
d S_{E}=2 \pi R_{E} \dot{R}_{E} d t=\frac{2 \pi R_{A}}{(1-\epsilon)^{2}} \epsilon d t
$$

and using the Hawking temperature for the event horizon we obtain

$$
T_{E} d S_{E}=\epsilon d t /(1-\epsilon)
$$

We do not obtain the first law with the above definitions since the energy income is larger by a factor $1 /(1-\epsilon)$.

One may argue that the temperature of the bath should be the same using the event and apparent horizon, which should be the local effect on the observer. If we choose $T_{A}$, the temperature on the apparent horizon, which is larger exactly by a factor $1 /(1-\epsilon)$, the difference $1-\epsilon$ disappears and the first law $-d E=T d S$ looks correct in this form.

But the problem is not solved. If we extend the discussion to the time-dependent equation of state DE, even if we use the local temperature on the apparent horizon, we shall see that the first law of thermodynamics still cannot be rescued on the event horizon, with the usual definitions of the temperature and entropy.

\section{B. Accelerating universes driven by DE with time-dependent equation of state.}

We shall use the holographic DE model [21]. The generalization of this model by considering interaction between DE and Dark matter (DM) has recently been discussed in [5]. For simplicity, we neglect the interaction between DE and DM for the moment, but the result below also holds if we include the interaction.

The event horizon in this case is given by the expression $R_{E}=a \int_{a}^{\infty} d a /\left(H a^{2}\right)=c /\left(\sqrt{\Omega_{D}} H\right)$. The last equality was gotten by considering the holographic DE $\rho_{D}=\Omega_{D} 3 H^{2}=3 c^{2} R_{E}^{-2}$ [21]. The apparent horizon is $R_{A}=1 / H$. The relation between apparent and event horizon is $R_{A} / R_{E}=$ $\sqrt{\Omega_{D}} / c$ and the apparent horizon is in general smaller than the event horizon. If $c=1$ and $\Omega_{D}=1$ both horizons are the same, $R_{E}=R_{A}$. 21.

Neglecting the interation between the DE and DM, the evolution of the DE was obtained as

$$
\Omega_{D}^{\prime}=\Omega_{D}^{2}\left(1-\Omega_{D}\right)\left[\frac{1}{\Omega_{D}}+\frac{2}{c \sqrt{\Omega_{D}}}\right]
$$

and the equation of state of the DE has the form

$$
w_{D}=-1 / 3-2 \sqrt{\Omega_{D}} /(3 c)
$$


With the evolution of the DE, $w_{D}$ changes with cosmological time.

We now examine how much the horizon will change over one Hubble time. For the apparent horizon

$$
t_{H} \frac{\dot{R_{A}}}{R_{A}}=a H \frac{d\left(H^{-1}\right)}{d a}=H \frac{d H^{-1}}{d x},
$$

where $x=\ln a$. From the Friedmann equation, we have $1-\Omega_{D}=\Omega_{m}=\rho_{m} /\left(3 H^{2}\right)=$ $\Omega_{m 0} H_{0}^{2} H^{-2} a^{-3}$, therefore $H^{-1}=a^{3 / 2} \sqrt{1-\Omega_{D}} /\left(H_{0} \sqrt{\Omega_{m 0}}\right)$. Eq (10) can be rewritten as

$$
t_{H} \frac{\dot{R_{A}}}{R_{A}}=3 / 2-\Omega_{D}^{\prime} /\left(2\left(1-\Omega_{D}\right)\right) .
$$

Considering the evolution of the DE, Eq.(8), we have

$$
t_{H} \frac{\dot{R_{A}}}{R_{A}}=3 / 2-\Omega_{D} / 2-\Omega_{D}^{3 / 2} / c
$$

We now calculate the change of the event horizon, which can be obtained from

$$
t_{H} \frac{\dot{R_{E}}}{R_{E}}=\frac{3}{2}-\frac{\Omega_{D}^{\prime}}{2 \Omega_{D}\left(1-\Omega_{D}\right)}=1-\frac{\sqrt{\Omega_{D}}}{c}
$$

We learn neither the apparent horizon nor the event horizon change significantly over one hubble scale. This can be seen from Fig. 1. Actually, the event horizon changes less than the Hubble horizon. The equilibrium thermodynamics still can be applied here. As originally suggested for black holes, the temperature and entropy on the horizon are described in Eq. (2).

Now we start to investigate the first law of thermodynamics on different horizons. For the apparent horizon, the total amount of energy crossing the apparent horizon during $d t$ is

$$
-d E=4 \pi R_{A}^{2} \rho(1+w) d t=3(1+w) / 2 d t
$$

Employing $w=\frac{P_{D}}{\rho}=w_{D} \rho_{D} /\left(3 H^{2}\right)=w_{D} \Omega_{D}=\left(-1 / 3-2 \sqrt{\Omega_{D}} /(3 c)\right) \Omega_{D}$, we get

$$
-d E=\left(3 / 2-\Omega_{D} / 2-\Omega_{D}^{3 / 2} / c\right) d t
$$

On the other hand, using the definitions of temperature on the horizon and the holographic entropy we get

$$
T_{A} d S_{A}=\dot{R}_{A} d t=H \frac{d H^{-1}}{d x} d t=\left[3 / 2-\Omega_{D} / 2-\Omega_{D}^{3 / 2} / c\right] d t .
$$

Thus on the apparent horizon, the first law, $-d E=T_{A} d S_{A}$, is confirmed, with the above definitions.

We now extend the discussion to the event horizon. The energy flow through the event horizon reads

$$
-d E=4 \pi R_{E}^{2} \rho(1+w) d t=\frac{3 c^{2}(1+w)}{2 \Omega_{D}} d t=\frac{c^{2}\left(3 / 2-\Omega_{D} / 2-\Omega^{3 / 2} / c\right)}{\Omega_{D}} d t .
$$




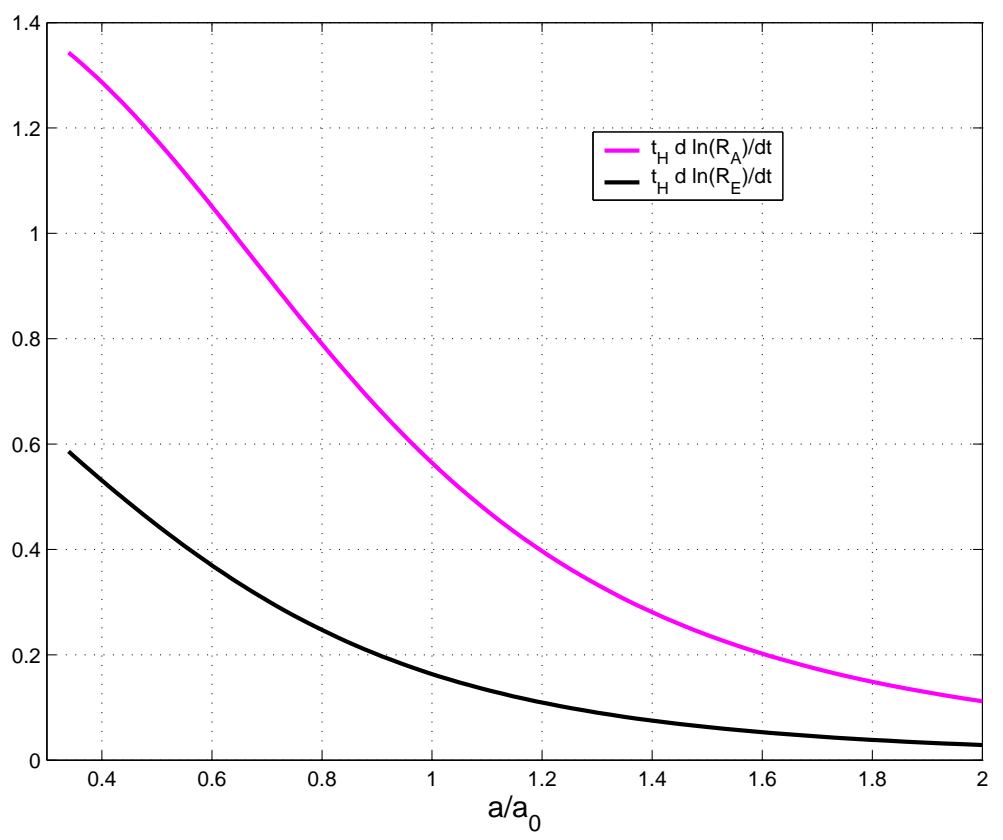

Figure 1: The rate changes of the apparent horizon and the event horizon over one Hubble scale with $\Omega_{D 0}=0.7$ and $c=1$.

To examine the first law, let us compute $T_{E} d S_{E}=\dot{R_{E}} d t$. Using

$$
\dot{R_{E}}=\frac{d}{d t}\left(\frac{c}{\sqrt{\Omega_{D}} H}\right)=H \frac{d}{d x}\left(\frac{c}{\sqrt{\Omega_{D}} H}\right)=\frac{3 c}{2 \sqrt{\Omega_{D}}}-\frac{c \Omega_{D}^{\prime}}{2 \Omega_{D}^{3 / 2}\left(1-\Omega_{D}\right)}
$$

and (8), we have $\dot{R_{E}}=c / \sqrt{\Omega_{D}}-1$.

We thus see that the first law cannot be satisfied in this form. It cannot be rescued even by using the same temperature of the bath on the apparent horizon, $T=1 /\left(2 \pi R_{A}\right)$, as we could have done in the Q-space, since

$$
T_{A} d S_{E}=\frac{R_{E}}{R_{A}} \dot{R_{E}} d t=\frac{c}{\sqrt{\Omega_{D}}} \dot{R_{E}} d t=\left[\frac{c^{2}}{\Omega_{D}}-\frac{c}{\sqrt{\Omega_{D}}}\right] d t
$$

Therefore, the first law of thermodynamics cannot hold at the event horizon with the usual definition of entropy and temperature!!

Although the dynamical difference between the apparent horizon and the event horizon is not large, the difference of their thermodynamical properties really is. In the de Sitter universe, this problem was hidden, since in that case the event horizon and the apparent horizon were degenerate. This problem becomes especially sharp with a dynamical equation of state. One reason could be that the first law may only apply to variations between nearby states of local thermodynamic equilibrium, while the event horizon reflects the global properties of spacetimes. At the event 
horizon, maybe the temperature and the entropy are ill-defined. In the nonstatic spacetime, the horizon thermodynamics are not simple, the notion of the surface gravity maybe ill-defined as argued in the study of the quasi-dS geometry in the inflationary universe [22] and the definition of temperature and entropy by means of the relations found by Bekenstein and Hawking are possibly wrong.

\section{ENTROPY AND THE SECOND LAW OF THERMODYNAMICS}

We will study the entropy enveloped by cosmological horizons. To exhibit the entropy of the accelerating universe with DE equation of state $w_{D}<-1$ together with $w_{D}>-1$ cases, we use our interacting holographic DE model [5].

The entropy of the universe inside the horizon can be related to its energy and pressure in the horizon by Gibb's equation [10]

$$
T d S=d E+P d V
$$

For the apparent horizon, considering $V=4 \pi R_{A}^{3} / 3, E=4 \pi \rho R_{A}^{3} / 3=R_{A} / 2, P=w \rho=$ $w_{D} \Omega_{D} 3 H^{2} /(8 \pi)$, we have

$$
T d S=d R_{A} / 2+(3 / 2) w_{D} \Omega_{D} d R_{A}
$$

Using $T=1 /\left(2 \pi R_{A}\right)$, which is the only temperature scale we have at our disposal, we get

$$
d S=\pi\left(1+3 w_{D} \Omega_{D}\right) R_{A} d R_{A} .
$$

Thus we see that the entropy enveloped by the apparent horizon is $S \sim R_{A}^{2}$.

Now we need to solve the equation to see the evolution of the entropy. Since $R_{A} d R_{A}=$ $R_{A} \frac{d R_{A}}{d x} d x=-\frac{1}{H^{3}} \frac{d H}{d x} d x$, we have

$$
\frac{d S}{d x}=-\pi\left(1+3 w_{D} \Omega_{D}\right) H^{-3} \frac{d H}{d x}=-\pi\left(1-3 b^{2}-\Omega_{D}-2 \Omega_{D}^{3 / 2} / c\right) H^{-3} \frac{d H}{d x}=-2 \pi q H^{-3} \frac{d H}{d x}
$$

where we have used the evolution of the DE in our interacting holographic DE model [5]

$$
\frac{\Omega_{D}^{\prime}}{\Omega_{D}}=1-3 b^{2}-\Omega_{D}-\frac{2 \Omega_{D}^{3 / 2}}{c}+\frac{2 \sqrt{\Omega_{D}}}{c}
$$

and the equation of state of the DE

$$
w_{D}=-\frac{\Omega_{D}^{\prime}}{3 \Omega_{D}\left(1-\Omega_{D}\right)}-\frac{b^{2}}{\Omega_{D}\left(1-\Omega_{D}\right)}=-\frac{1}{3}-\frac{2}{3} \frac{\sqrt{\Omega_{D}}}{c}-\frac{b^{2}}{\Omega_{D}} .
$$


Above, $b^{2}$ is the coupling between DE and DM. The evolution of the Hubble parameter in our model was described as

$$
\frac{d \ln H}{d x}=-3 / 2+3 b^{2} / 2+\Omega_{D} / 2+\Omega_{D}^{3 / 2} / c=-\left(3-3 b^{2}-\Omega_{D}-2 \Omega_{D}^{3 / 2} / c\right) / 2 \quad .
$$

In (23) $q$ is the deceleration parameter, given by

$$
q=-\dot{H} / H^{2}-1=-3\left[-1-w_{D}-\left(1-\Omega_{D}\right) / \Omega_{D}\right] \Omega_{D} / 2-1=\left(1-3 b^{2}-\Omega_{D}-2 \Omega_{D}^{3 / 2} / c\right) / 2
$$

To accommodate the transition of the DE equation of state from $w_{D}>-1$ to $w_{D}<-1$ at recent stage as indicated by observations [4], we constrained our model parameters $c$ from holography in the range $\sqrt{\Omega_{D}}<c<1.255$ and $b^{2}$ of the coupling between DE and DM within the range $1.4(1-\sqrt{0.7} / c) / 3<b^{2}<8 c^{2} / 81[\underline{5}]$. Within these parameter space, we have $\Omega_{D}^{\prime}>0$ and $H^{\prime}<0$. At early stage, $\Omega_{D}$ is small and the universe was in the deceleration era with $q>0$, so we obtain $d S / d x>0$. However at late time, DE starts to dominate, the universe evolved in an accelerated expansion with $q<0$, thus we get $d S / d x<0$. Therefore the entropy of the universe enveloped by the apparent horizon increased first and then started to decrease. The entropy will decrease to a negative value at a late era of the universe when it is dominated by phantom fields, which is consistent with those observed in 9] 10]. The behavior of this entropy is shown in the dotted line in Fig 2.
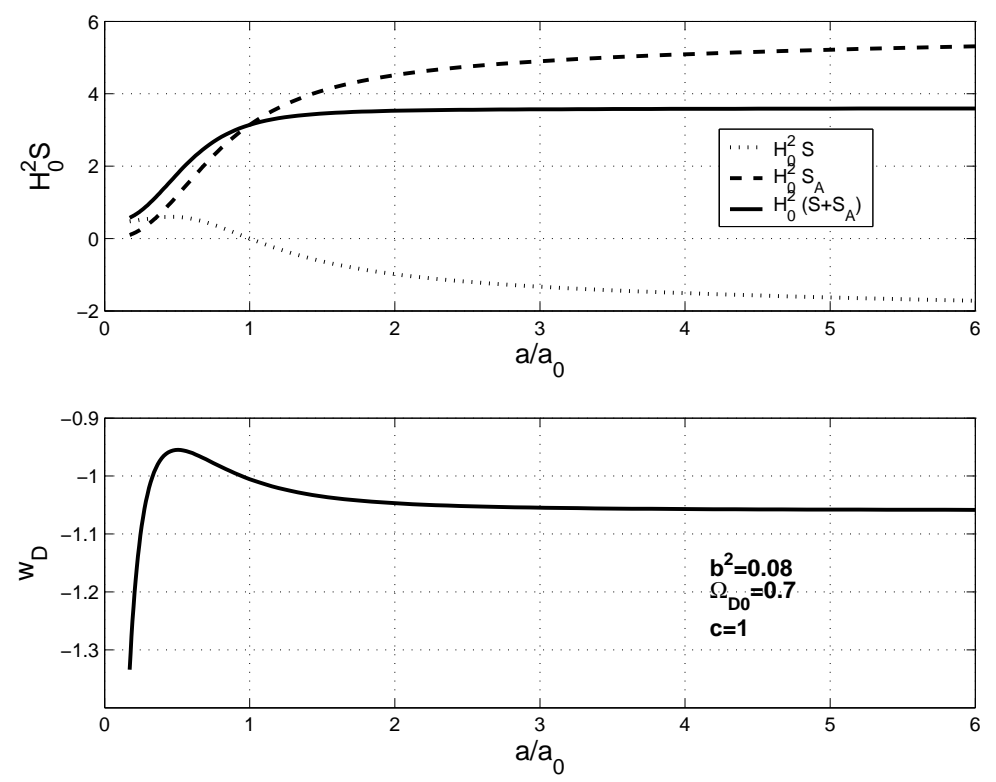

Figure 2: The evolution of entropies and $\omega_{D}$ with the parameters $b^{2}=0.08$ and $c=1$ and the initial conditions $\Omega_{D 0}=0.7$ and $H^{2} S_{0}=10^{-30}$. 
In addition to the entropy in the universe, there is a horizon entropy of the apparent horizon $S_{A}=\pi H^{-2}$. The evolution of this horizon entropy is shown in the dashed line in Fig.2. At the early stage we see that $S>S_{A}$, which is in violation of the holographic principle. This tells us that

although our interacting holographic DE model 5] is sucessful to describe the late time accelerating universe with the transition equation of state of DE, this model is not appropriate to describe the early deceleration of the universe.

In order to check the generalized second law (GSL) of gravitational thermodynamics, we have also plotted the evoluation of $S+S_{A}$, the solid line in Fig.2. It is easy to see that entropy of matter and fluids inside the apparent horizon plus the entropy of the apparent horizon do not decrease with time. Thus the GSL is respected in the universe enveloped by the apparent horizon.

It is of interest to examine the GSL in the universe enveloped by the cosmological event horizon. The evolution of the entropy inside the event horizon can be gotten similarly as that of the apparent horizon

$$
\frac{d S}{d x}=-\pi c^{4}\left(1+3 w_{D} \Omega_{D}\right) H^{-3} \Omega_{D}^{-2} \frac{d H}{d x}-\frac{3 \pi}{2} c^{4}\left(1+w_{D} \Omega_{D}\right) H^{-2} \Omega_{D}^{-3} \frac{d \Omega_{D}}{d x} \quad,
$$

while the evolution of the geometric entropy on the event horizon reads

$$
\frac{d S_{E}}{d x}=-2 \pi c^{2} H^{-3} \Omega_{D}^{-1} \frac{d H}{d x}-\pi c^{2} H^{-2} \Omega_{D}^{-2} \frac{d \Omega_{D}}{d x}
$$

We thus find

$$
\frac{d\left(S+S_{E}\right)}{d x}=\frac{\pi}{2} c^{4} H^{-2} \Omega_{D}^{-2}[A B-C D]
$$

where $A=2 q+2 \Omega_{D} / c^{2}, B=2+2 q, C=2 q+2 \sqrt{\Omega_{D}} / c, D=2+2 q+2 \Omega_{D} / c^{2}$. Since in our model it is required that $\sqrt{\Omega_{D}}<c$, we have $A<C$ and $B<D$. Thus, for the universe enveloped by the cosmological event horizon we have $d\left(S+S_{E}\right) / d x<0$ and the GSL breaks down, with the usual definitions of entropy for the boundary.

\section{CONCLUSIONS AND DISCUSSIONS}

The apparent horizon of the universe always exists and the thermodynamical properties related to the apparent horizon has been studied by several authors, including in a quasi-dS geometry of inflationary universe [22] and a late time accelerating Q-space [7]. In this paper we have extended the investigation to an accelerated expanding universe driven by $\mathrm{DE}$ of time-dependent equation of state. We have confirmed that the first law of thermodynamics still holds for this dynamic 
apparent horizon. Besides we have also examined the GSL of gravitational thermodynamics of the accelerating universe driven by the interacting holographic DE [5] with the transition equation of state. The entropy of matter together with fluids inside the apparent horizon plus the entropy of the apparent horizon always increase with time, which shows that the GSL is protected in the accelerating universe enveloped by the apparent horizon.

In the usual standard big bang model a cosmological event horizon is absent. The thermodynamics related to the cosmological event horizon was studied in the dS space [16], however in dS space, the cosmological event horizon and apparent horizon degenerate. In a general accelerating universe driven by DE with equation of state $w_{D} \neq-1$, the cosmological event horizon separates from that of the apparent horizon. In this paper we have tried to apply the usual definition of the temperature and entropy as that of the apparent horizon to the cosmological event horizon and examine the first and the second laws of thermodynamics. In contrast to the case of the aparent horizon, we are surprised to find that both the first and second law of thermodynamics break down if we consider the universe to be enveloped by the event horizon with the usual definitions of entropy and temperature. The break down of the first law could be attributed to the possibility that the first law may only apply to variations between nearby states of local thermodynamic equilibrium, while the event horizon reflects the global spacetime properties. Besides in the dynamic spacetime, the horizon thermodynamics is not as simple as that of the static spacetime. The event horizon and apparent horizon are in general different surfaces. The definition of thermodynamical quantities on the cosmological event horizon in the nonstatic universe are probably ill-defined. This was first argued in the quasi-dS geometry of the inflationary spacetime [22].

Furthermore we would like to point out that the apparent horizon serving as a specific surface was observed by Bousso in studying the covariant entropy bound 23]. The apparent horizon was singled out as the largest surface whose interior can be treated as a Bekenstein system, which satisfies the Bekenstein's entropy/mass bound $S \leq 2 \pi R E$ and Bekenstein's entropy/area bound $S \leq A / 4$. In the region surrounded by the surface outside the apparent horizon, one could satisfy Bekenstein entropy/mass bound but break Bekenstein entropy/area bound, which indicates a breakdown of the treatment of the enclosed region as a Bekenstein system. Since Bekenstein bounds are universal, all gravitational stable spacial regions with weak self-gravity should satisfy Bekenstein bounds and the corresponding thermodynamical system is a Bekenstein system. This should also be true in cosmological situations. For the dS situation, since the cosmological event horizon coincides with the apparent horizon, the region enclosed by the cosmological horizon satisfies Bekenstein bounds. However for the accelerating universe driven by DE with $w_{D} \neq-1$, 
from our equations (5) and (17) above, for example, we see that at the event horizon $d E>d R_{E}$, or $E>R_{E}$. Although the Bekenstein entropy/mass bound can be satisfied, the Bekenstein entropy/area bound is violated, since $S=2 \pi E R_{E}>\pi R_{E}^{2}$. Thus the thermodynamic system outside the apparent horizon is no longer a Bekenstein system and the usual thermodynamic description breaks down.

To our knowledge, this thermodynamical problem related to the event horizon in the accelerating universe driven by the DE has not been found before. The solution of this problem requires further knowledge, which we do not have at disposal at this moment.

\section{Acknowledgments}

This work was partially supported by NNSF of China, Ministry of Education of China, Ministry of Science and Technology of China under grant No. NKBRSFG19990754 and Shanghai Education Commission. Y. Gong's work was supported by NNSFC under grant No. 10447008, CSTC under grant No. 2004BB8601, CQUPT under grant No. A2004-05 and SRF for ROCS, State Education Ministry. E. Abdalla's work was partially supported by FAPESP and CNPQ, Brazil. E.A. wishes to thank G. Matsas, S. Salinas and W. Wreszinski for discussions.

[1] A.G. Riess, et al., Astron. J. 116 (1998) 1009; S. Perlmutter, et al., Astrophys. J. 517 (1999) 565; S. Perlmutter, et al., Astrophys. J. 598 (2003) 102; P. de Bernardis, et al., Nature 404 (2000) 955.

[2] G. Hinshaw et al, Astrophys. J. Suppl. 148 (2003) 135; L. Page et al, Astrophys. J. Suppl. 148 (2003) 39; D.N. Spergel et al. Astrophys. J. Suppl. 148 (2003) 175.

[3] T. Padmanabhan, Phys. Rep. 380235 (2003); P. J. E.Peebles, B. Ratra, Rev. Mod. Phys. 75559 (2003); V. Sahni, Lect. Notes Phys. 653 (2004) 141 astro-ph/0403324

[4] U. Alam, V. Sahni and A. A. Starobinsky, JCAP 0406 (2004) 008; D. Huterer and A. Cooray, Phys. Rev. D 71 (2005) 023506; Y. Wang and M. Tegmark, astro-ph/0501351

[5] B. Wang, Y. G. Gong and E. Abdalla, hep-th/0506069, Phys. Lett. B 624 (2005) 141.

[6] Bin Wang, C. Y. Lin and E. Abdalla hep-th/0509107 Bin Wang, E. Abdalla and Ru Keng Su Phys. Lett. B611 (2005).

[7] R. Bousso, Phys. Rev. D71 (2005) 064024, hep-th/0412197.

[8] J. A. S. Lima and J. S. Alcaniz, Phys. Lett. B600 (2004) 191.

[9] I. Brevik, S. Nojiri, S. D. Odintsov and L. Vanzo, Phys. Rev. D70 (2004) 043520.

[10] G. Izquierdo and D. Pavon, astro-ph/0505601

[11] J. Bekenstein Phys. Rev. D7 (1973) 2333. 
[12] S. Hawking Commun. Math. Phys. 43 (1975) 199.

[13] T. Jacobson Phys. Rev. Lett. 75 (1995) 1260.

[14] Gerard 't Hooft, in Salamfest 0284-296 (1993) QCD161:C512, gr-qc/9310026 Leonard Susskind, J. Math. Phys. 36 (1995) 6377.

[15] Ivo Savonije and Erik P. Verlinde, Phys. Lett. B507 (2001) 305, Erik P. Verlinde, hep-th/0008140

[16] G. W. Gibbons and S. W. Hawking, Phys. Rev. D15 (1977) 2738.

[17] A. Strominger J. High Energy Phys. 10 (2001) 034.

[18] E. Abdalla, K.H.C. Castello-Branco and A. Lima-Santos, Phys. Rev. D66 (2002) 104018, hep-th/0208065 Elcio Abdalla, Bin Wang, A. Lima-Santos and W.G. Qiu, Phys. Lett. B538 (2002) 435 hep-th/0204030

[19] Bert Schroer, hep-th/0507038

[20] R. G. Cai and S. P. Kim, JHEP 0502 (2005) 050, hep-th/0501055.

[21] M. Li, Phys. Lett. B603 (2004) 1.

[22] A. Frolov and L. Kofman, JCAP 0305 (2003) 009, hep-th/0212327

[23] Raphael Bousso, JHEP 9907 (1999) 004, hep-th/9905177 